\title{
Vacuum fluctuations of the supersymmetric field in curved background
}

\author{
Neven Bilić;, Silvije Domazet†, Branko Guberina ${ }^{\ddagger}$ \\ Rudjer Bošković Institute, POB 180, HR-10002 Zagreb, Croatia
}

January 18, 2012

\begin{abstract}
We study a supersymmetric model in curved background spacetime. We calculate the effective action and the vacuum expectation value of the energy momentum tensor using a covariant regularization procedure. A soft supersymmetry breaking induces a nonzero contribution to the vacuum energy density and pressure. Assuming the presence of a cosmic fluid in addition to the vacuum fluctuations of the supersymmetric field an effective equation of state is derived in a self-consistent approach at one loop order. The net effect of the vacuum fluctuations of the supersymmetric fields in the leading adiabatic order is a renormalization of the Newton and cosmological constants.
\end{abstract}

\section{Introduction}

Observational evidence for an accelerating expansion [1, 2, 3] implies that the vacuum energy density dominates the total energy density today. The vacuum energy or cosmological constant (CC) which was introduced $a d$-hoc in the Einstein-Hilbert action is related to the vacuum energy density of matter fields. The main $\mathrm{CC}$ problem is that the vacuum energy contribution estimated in quantum field theory is much larger than the observed value. In general, the result depends quartically on a quantity $\Lambda_{\text {cut }}$ that represents the UV momentum cutoff, i.e., the vacuum energy density goes as $\Lambda_{\text {cut }}^{4}$. If $\Lambda_{\text {cut }}$ is of the order of the Planck mass, the estimated vacuum energy density is by about 120 orders of magnitude larger than the value required by astrophysical and cosmological observations [4]. This term, if really there, would be disastrous even if the cutoff were in the mass range of the standard model of particle physics.

The above mentioned estimate was based on field theory in flat spacetime in which one can easily solve the problem by redefining the vacuum energy. Since the energy is defined up to an arbitrary additive constant one can subtract the divergent contributions and make

*Electronic mail: bilic@thphys.irb.hr

${ }^{\dagger}$ Electronic mail: sdomazet@irb.hr

†Electronic mail: guberina@thphys.irb.hr 
the vacuum energy exactly zero 1. However, in curved spacetime this procedure cannot be performed because the energy is a source of the gravitational field and adding or subtracting (even constant) energy changes the spacetime geometry. In spite of that, the quantum field theory in curved spacetime is renormalizable provided higher derivative terms in addition to the Einstein-Hilbert term with a cosmological constant are introduced at the classical level. In what follows we use the low energy formulation of quantum field theory in curved spacetime [5, 6]. The theory is described by the gauged matter Lagrangian in addition to the Einstein-Hilbert action.

Phenomenologically, it is desirable to have the vacuum energy exactly zero or comparable to the tiny value of the critical density of the universe. To achieve that, one must either kill the flat spacetime contribution simply by fiat or invent a symmetry principle that forbids a nonzero vacuum energy. Such a principle is indeed provided by supersymmetry [7]. In field theory with exact supersymmetry, the contributions of fermions and bosons to vacuum energy precisely cancel [8].

In references [9, 10] a residual quadratic contribution of the form $H^{2} \Lambda_{\text {cut }}^{2}$ has been found after canceling the flat spacetime parts in the SUSY limit. Such a contribution is phenomenologically acceptable owing to the fact that the present value of the CC density is of the same order, if $\Lambda_{\text {cut }}$ is taken to be of the order of the Planck mass. Such quadratic contribution was also found in recent papers [11, 12] and in some earlier papers [13, 14] in a different context. In particular, the work [11] presents a similar calculation of the zero-point energy using only a massless boson field and obtains two types of contributions: the quartic type $\Lambda_{\text {cut }}^{4}$ and the quadratic part $H^{2} \Lambda_{\text {cut }}^{2}$. Then, the quartic contributions to $\mathrm{CC}$ was canceled on the basis of the procedure used previously in the literature with the so-called ADM mass. In [9, 10] it has been demonstrated that in a supersymmetric world a cancellation by fiat is unnecessary because the cancellation between bosons and fermions of all (not only quartically divergent) flat-spacetime contributions is naturally provided by supersymmetry. However, in real world where SUSY is broken this cancellation will affect only the quartic contribution. If SUSY is broken at scales $m_{\text {SUSY }}$ the dominant flat-spacetime contributions will be the quadratically divergent contribution of the type $m_{\mathrm{SUSY}}^{2} \Lambda_{\text {cut }}^{2}$ and the mass terms of the type $m_{\mathrm{SUSY}}^{4}$. These flat-spacetime contributions will of course be present in nonflat spacetime too.

Another important point of both papers [10] and [11] is that the vacuum fluctuations cannot be interpreted as a part of $\mathrm{CC}$ because the vacuum fluctuations do not yield the equation of state $p=-\rho$, as a consequence of the energy momentum tensor not having a $\mathrm{CC}$ form. This behavior was already observed in flat space time if a three-dimensional cutoff regularization was employed [15, 16, 17]. A possible way out has recently been suggested by Maggiore et al [18, 19]. If the regularization scheme breaks general covariance, one must also allow for noncovariant counterterms, and these can be chosen so to obtain fully covariant results for the renormalized energy density and pressure. For example, since the quartic term in the bare energy density and pressure does not satisfy $p=-\rho$ (the vacuum fluctuations of a minimally coupled scalar field gives $p=\rho / 3$ ), one may introduce appropriate noncovariant

\footnotetext{
${ }^{1}$ Unfortunately, the cancellation of the divergent contributions does not solve the cosmological constant problem. The remaining finite contributions of the type $m^{4}$ in a realistic field theory are still much larger then the observed vacuum density. Besides, all such contributions coming from different sectors of the standard model of particle physics should somehow conspire in order to reproduce the observe tiny value of the vacuum energy density. Extreme fine tuning is needed for this to happen.
} 
counterterms so that the renormalized quantities satisfy $p_{\text {ren }}=-\rho_{\text {ren }}$. The explicit form of these counterterms, and a detailed discussion of the above issues, has been given in [19] (elaborating on results of [18]).

Using a three-dimensional cutoff $\Lambda_{\text {cut }}$ procedure is perhaps not the most elegant way to regularize a quantum field theory. First, the three-dimensional cutoff violates Lorentz invariance and, as a result, the flat space time terms in the energy density and pressure do not satisfy the $\mathrm{CC}$ equation of state. This is not a problem for a model with an exact supersymmetry since these terms cancel anyway. However, one cannot be sure that the residual EOS coming from the dominant contribution of the type $H^{2} \Lambda_{\text {cut }}^{2}$ is robust, i.e. one does not know how much it is scheme dependent. Therefore, it would be desirable to use an explicitly covariant scheme to regularize the integrals. A covariant regularization in flat space time should yield the vacuum energy momentum tensor of the form $T_{\mu \nu}=\rho \eta_{\mu \nu}$. Naively, in curved spacetime one would generalize this to the CC form $T_{\mu \nu}=\rho g_{\mu \nu}$. However, since a curved geometry involves the Riemann tensor and its covariant derivatives we may expect the energy momentum tensor at linear curvature order to be of the form $T_{\mu \nu}=(\alpha+\beta R) g_{\mu \nu}+\gamma R_{\mu \nu}$ where $\alpha, \beta$, and $\gamma$ are constants that do not depend on curvature.

The main motivation for this paper is to investigate the fate of vacuum energy when an unbroken supersymmetric model is embedded in a general curved spacetime. We propose an approach based on the effective action. We calculate one loop contributions to the effective and we regularize divergences using a covariant UV cutoff. Our approach is similar to Sobreira et al [20] who calculated the scalar and fermion one loop contributions to the effective potential. From the effective action we derive the effects of the vacuum energy fluctuations of the supersymmetric fields on the expansion of the universe, specifically on the cosmological constant. With a cutoff scale of the order of the Planck mass $m_{\mathrm{Pl}}$ the contribution of the supersymmetric field fluctuations is found to be of the same order of magnitude as that of the cosmic fluid. For example, in the case of CC, i.e., in the de Sitter background with the expansion parameter $H$, the contribution is of the order $H^{2} m_{\mathrm{Pl}}^{2}$ no fine-tuning is needed.

Unlike in flat spacetime, the vacuum energy density turns out to be nonzero depending on background metric. This type of "soft" supersymmetry breaking is similar to the supersymmetry breaking at finite temperature where the Fermi-Bose degeneracy is lifted by quantum statistics ([21] and references therein). In addition to the supersymmetric field we assume the presence of a cosmic fluid obeying the equation of state of the general form $p=p(\rho)$ so that that the global geometry is determined by a combined effect of both the cosmic fluid and vacuum fluctuations of the supersymmetric field.

The remainder of the paper is organized as follows. In section 2 we introduce a supersymmetric model in an arbitrary curved background. The calculations of the effective action at one loop order are presented in section 3 . In section 4 we derive the vacuum expectation value of the energy momentum tensor and discuss the effective equation of state. Concluding remarks are given in section 5 . 


\section{The model}

We consider a Wess-Zumino supersymmetric model with $N$ species and calculate the energy density of vacuum fluctuations in arbitrary curved background. Our notations follow [10]. In general, the supersymmetric Lagrangian for $N$ chiral superfields has the form [22]

$$
\mathcal{L}_{N}=\left.\sum_{i} \Phi_{i}^{\dagger} \Phi_{i}\right|_{D}+\left.W(\Phi)\right|_{F}+\text { h.c. }
$$

where the index $i$ distinguishes the various left chiral superfields $\Phi_{i}$ The quantity $W(\Phi)$ denotes the superpotential for which we take

$$
W(\Phi)=\sum_{i}\left(\frac{m_{i}}{2} \Phi_{i}^{2}+\frac{\lambda}{3} \Phi_{i}^{3}\right) .
$$

so that the Lagrangian (1) is just a sum of $N$ chiral Lagrangians $\mathcal{L}_{i}$ of each species. For simplicity, from now on we suppress the dependence on $i$. Assuming a curved background spacetime geometry with metric $g_{\mu \nu}$ and eliminating auxiliary fields by equations of motion, the Lagrangian for each species takes the form

$$
\begin{aligned}
\mathcal{L}= & g^{\mu \nu} \phi_{, \mu}^{\dagger} \phi_{, \nu}-V(\phi)+\frac{i}{4}\left(\bar{\Psi} \tilde{\gamma}^{\mu} \Psi_{; \mu}-\bar{\Psi}_{; \mu} \tilde{\gamma}^{\mu} \Psi\right)-\frac{1}{2} m \bar{\Psi} \Psi \\
& -\frac{\lambda}{2} \bar{\Psi}\left(1-\gamma_{5}\right) \Psi \phi-\frac{\lambda}{2} \bar{\Psi}\left(1+\gamma_{5}\right) \Psi \phi^{\dagger}
\end{aligned}
$$

with

$$
V(\phi)=\left|m \phi+\lambda \phi^{2}\right|^{2}+\xi R|\phi|^{2}
$$

Here, $\phi$ and $\Psi$ are the complex scalar and the Majorana spinor fields, respectively, and $\tilde{\gamma}^{\mu}$ are the curved spacetime gamma matrices [5]. For completeness, we included in the scalar field potential (4) the nonminimal coupling term $\xi R|\phi|^{2}$ of the scalar field to the scalar curvature $R$. The term is needed for renormalization because, even if set $\xi=0$ in (3), the loop corrections would generate in the effective action a term of this type [5, 23].

In the chiral $(m \rightarrow 0)$ limit, the Lagrangian (3) becomes invariant under the chiral U(1) transformation

$$
\begin{aligned}
\phi & \rightarrow e^{i 2 \alpha} \phi \\
\left(1-\gamma_{5}\right) \Psi & \rightarrow e^{-i \alpha}\left(1-\gamma_{5}\right) \Psi \\
\left(1+\gamma_{5}\right) \Psi & \rightarrow e^{i \alpha}\left(1+\gamma_{5}\right) \Psi
\end{aligned}
$$

This invariance reflects the $R$-invariance of the cubic superpotential [24]. The action may be written as

$$
S=\int d^{4} x \sqrt{-g}\left(\mathcal{L}_{\mathrm{B}}+\mathcal{L}_{\mathrm{F}}\right),
$$

where $\mathcal{L}_{\mathrm{B}}$ and $\mathcal{L}_{\mathrm{F}}$ are the boson and fermion Lagrangians, respectively. Using

$$
\phi=\frac{1}{\sqrt{2}}(\sigma+i \pi)
$$


the Lagrangian for a complex scalar field $\phi$ may be expressed as a Lagrangians for two real fields $\sigma$ and $\pi$. Then, the potential (4) becomes

$$
V(\sigma, \pi)=\frac{m^{2}+\xi R}{2}\left(\sigma^{2}+\pi^{2}\right)+\frac{\lambda^{2}}{4}\left(\sigma^{2}+\pi^{2}\right)^{2}+\frac{m \lambda}{\sqrt{2}} \sigma\left(\sigma^{2}+\pi^{2}\right) .
$$

Variation of (8) with respect to $\bar{\Psi}$ yields the Dirac equation in curved spacetime:

$$
i \tilde{\gamma}^{\mu} \Psi_{; \mu}-\left(m+\sqrt{2} \lambda \sigma-i \sqrt{2} \lambda \pi \gamma_{5}\right) \Psi=0
$$

\section{Effective action}

In this section we derive the effective action in a general curved background defined by a metric $g_{\mu \nu}$ and the corresponding curvature tensor $R_{\mu \nu}$. We use the method described in [23, 25] based on the calculation of the Feynman propagator at one loop order. We introduce the background fields $\bar{\sigma}$ and $\bar{\pi}$ and redefine the fields

$$
\sigma \rightarrow \bar{\sigma}+\sigma ; \quad \pi \rightarrow \bar{\pi}+\pi
$$

The effective action at one loop order is given by [23]

$$
\Gamma[\bar{\sigma}, \bar{\pi}]=S^{(0)}[\bar{\sigma}, \bar{\pi}]-i \ln \int[d \sigma, d \pi, d \Psi] \exp \left(i S^{(2)}[\bar{\sigma}, \bar{\pi}, \sigma, \pi, \Psi]\right),
$$

where $[d \sigma, d \pi, d \Psi]$ denotes the measure of the functional integral, $S^{(0)}$ is the classical part of the action (8) and $S^{(2)}$ is the part of the action which is quadratic in quantum fields. The classical part is just the boson part of the action (8) in which the fields $\sigma$ and $\pi$ are replaced by $\bar{\sigma}$ and $\bar{\pi}$.

For the quadratic part we find

$$
S^{(2)}=\int d^{4} x \sqrt{-g}\left(\mathcal{L}_{\mathrm{B}}^{(2)}+\mathcal{L}_{\mathrm{F}}^{(2)}\right)
$$

where

$$
\mathcal{L}_{\mathrm{B}}^{(2)}=\frac{1}{2} g^{\mu \nu} \sigma_{, \mu} \sigma_{, \nu}+\frac{1}{2} g^{\mu \nu} \pi_{, \mu} \pi_{, \nu}-\frac{m_{\sigma}^{2}}{2} \sigma^{2}-\frac{m_{\pi}^{2}}{2} \pi^{2}-\frac{\xi}{2} R\left(\sigma^{2}+\pi^{2}\right)
$$

and

$$
\mathcal{L}_{\mathrm{F}}^{(2)}=\frac{i}{4}\left(\bar{\Psi} \tilde{\gamma}^{\mu} \Psi_{; \mu}-\bar{\Psi}_{; \mu} \tilde{\gamma}^{\mu} \Psi\right)-\frac{1}{2} m_{\mathrm{F}} \bar{\Psi} \Psi+\frac{i \lambda \bar{\pi}}{\sqrt{2}} \bar{\Psi} \gamma_{5} \Psi .
$$

The effective boson masses $m_{\sigma}^{2}$ and $m_{\pi}^{2}$, are the coefficients of the diagonalized quadratic form in $\sigma$ and $\pi$, and $m_{\mathrm{F}}$ is the effective fermion mass. We find

$$
m_{\sigma}^{2}=a+b ; \quad m_{\pi}^{2}=a-b ; \quad m_{\mathrm{F}}=m+\sqrt{2} \lambda \bar{\sigma}
$$

where

$$
a=m^{2}+2 \lambda^{2}\left(\bar{\sigma}^{2}+\bar{\pi}^{2}\right)+2 \sqrt{2} m \lambda \bar{\sigma}
$$

and

$$
b=\lambda \sqrt{\left(\bar{\sigma}^{2}+\bar{\pi}^{2}\right)\left[(\lambda \bar{\sigma}+\sqrt{2} m)^{2}+\lambda^{2} \bar{\pi}^{2}\right]} .
$$

In the following we calculate separately the contributions of the fermion and scalar fields. 


\subsection{Scalar fields}

Following Parker and Toms [23] the contribution of each scalar field may be calculated using the expression

$$
\Gamma_{s}=-\frac{i}{2} \int d^{4} x \sqrt{-g} \int^{m_{s}^{2}} d\left(m^{2}\right) \Delta(x, x),
$$

where the subscript $s$ stands for either $\sigma$ or $\pi$. The renormalized Feynman propagator

$$
\Delta\left(x, x^{\prime}\right)=-i\left\langle\varphi(x) \varphi\left(x^{\prime}\right)\right\rangle
$$

satisfies the equation

$$
\left(-\square-m^{2}-\xi R\right) \Delta\left(x, x^{\prime}\right)=(-g(x))^{-1 / 2} \delta\left(x-x^{\prime}\right),
$$

where $\square=g^{\mu \nu} \nabla_{\mu} \nabla_{\nu}$. In the coincidence limit, $x \rightarrow x^{\prime}$,

$$
\Delta(x, x)=\int \frac{d^{d} k}{(2 \pi)^{d}}\left[1+\sum_{j=1}^{\infty} \bar{f}_{j}\left(-\frac{\partial}{\partial m^{2}}\right)^{j}\right]\left[k^{2}-m^{2}-\left(\xi-\frac{1}{6}\right) R(x)\right]^{-1},
$$

where $k^{2}=\eta_{\mu \nu} k^{\mu} k^{\nu}$ and the coefficient $\bar{f}_{j}$ involve covariant terms formed from the Riemann tensor, its contractions and covariant derivatives [23]. It is important to note that the expansion (23), although obtained using the Riemann normal coordinates [26], is valid in a general coordinate system. Three remarks are in order. First, in the expansion of $\Delta$ increasingly high orders in curvature are compensated by increasingly high orders in $\left(k^{2}-\right.$ $\left.m^{2}\right)^{-1}$. Second, any Feynman diagram in flat spacetime is substituted in curved spacetime by an infinite set of diagrams. However, these diagrams have better UV convergences than the diagrams in flat Minkowski space. Third, if one starts with a multiplicatively renormalizable theory in Minkowski spacetime, then in curved spacetime the total number of divergent diagrams, at any given loop order, is finite. Hence, the locality of UV terms is secured [27].

The Feynman propagator (21) may be written as [28]

$$
\Delta\left(x, x^{\prime}\right)=(-g(x))^{-1 / 4} \bar{\Delta}\left(x, x^{\prime}\right),
$$

where the modified propagator $\bar{\Delta}$ satisfies

$$
\left(-\square-m^{2}-\xi R\right) \bar{\Delta}\left(x, x^{\prime}\right)=\delta\left(x-x^{\prime}\right) .
$$

In Riemann normal coordinates the modified propagator takes the form

$$
\bar{\Delta}\left(x, x^{\prime}\right)=\int \frac{d^{d} k}{(2 \pi)^{d}} e^{-i k\left(x-x^{\prime}\right)}\left[\frac{1}{k^{2}-m^{2}}+\left(\xi-\frac{1}{6}\right) \frac{R}{\left(k^{2}-m^{2}\right)^{2}}+\ldots\right] .
$$

where the ellipses denote the terms of higher curvature order. Here, $x^{\prime}$ is the origin of the Riemann normal coordinate system and $R$ is evaluated at the origin of these normal coordinates.

In the limit $x^{\prime} \rightarrow x$, the momentum integrals in (26) are divergent in 4 dimensions and must be regularized. Naively, one would expect that using a four-dimensional covariant cutoff 
automatically ensures the correct answer. Unfortunately, it is not so, as Ossola and Sirlin [15] demonstrated in flat spacetime. Even doing the regularization of zero point-energy of the scalar field one gets wrong signs of the vacuum energy density vs. pressure. The most elegant way to deal with divergencies seems to be dimensional regularization (DR) 29]. However, DR has its own obstacles. First, DR does not identify the quadratic divergencies which are important in any effective field theory. Loosing the track of quadratic divergencies may corrupt the Wilson's renormalization group method. Next, since DR is essentially a mass independent scheme, particle thresholds and particle decoupling is not properly described and one is forced to put them in theory by fiat. Ossola and Sirlin [15] showed that the Pauli-Villars regularization, although covariant, leads to unacceptable result: the vacuum energy density of a scalar field turns out to be negative. Besides, the quartic divergencies break the scale invariance of the free field theory in the massless limit.

Keeping all previously said in mind, we perform the calculations using the prescriptions of Cynolter and Lendvai [30]. Veltman has early noticed that a correct calculation of quadratic divergencies in $d=2-2(\epsilon-1)$ leads to a cutoff regularization based on the DR. Doing correctly all conditions, preserving the symmetries, and using the prescription

$$
l_{\mathrm{E} \mu} l_{\mathrm{E} \nu} \rightarrow \frac{1}{d} g_{\mu \nu} l_{\mathrm{E}}^{2}
$$

dictated by Lorenz invariance, with the parameter $d$ to be determined using the Euclidean four-dimensional momentum cutoff, a relation between the cutoff and the DR is obtained. Matching different powers of $\Lambda_{\text {cut }}$ and preserving the gauge invariance, the following prescription is proposed [30]

$$
\begin{aligned}
\frac{1}{d} \Lambda_{\text {cut }}^{2} & \rightarrow \frac{1}{2} \Lambda_{\text {cut }}^{2}, \\
\frac{1}{d} \ln \left(\frac{\Lambda_{\text {cut }}^{2}+m^{2}}{m^{2}}\right) & \rightarrow \frac{1}{4}\left(\ln \left(\frac{\Lambda_{\text {cut }}^{2}+m^{2}}{m^{2}}\right)+\frac{1}{2}\right), \\
\frac{1}{d} & \rightarrow \frac{1}{4} \text { for finite terms, }
\end{aligned}
$$

which yields

$$
\begin{gathered}
\int \frac{d^{d} k}{(2 \pi)^{d}} \frac{1}{k^{2}-m^{2}}=-\frac{i}{(4 \pi)^{2}}\left(\Lambda_{\mathrm{cut}}^{2}-m^{2} \ln \frac{\Lambda_{\mathrm{cut}}^{2}}{m^{2}}\right), \\
\int \frac{d^{d} k}{(2 \pi)^{d}} \frac{1}{\left(k^{2}-m^{2}\right)^{2}}=\frac{i}{(4 \pi)^{2}}\left(\ln \frac{\Lambda_{\mathrm{cut}}^{2}}{m^{2}}-1\right) .
\end{gathered}
$$

Evaluating the integral over $m^{2}$ in (20) we find

$$
\begin{aligned}
& \Gamma_{s}=\frac{1}{2(4 \pi)^{2}} \int d^{4} x \sqrt{-g}\left[\frac{m_{s}^{4}}{4}-m_{s}^{2} \Lambda_{\text {cut }}^{2}+m_{s}^{2}\left(\frac{m_{s}^{2}}{2}+\left(\xi-\frac{1}{6}\right) R\right) \ln \frac{\Lambda_{\text {cut }}^{2}}{m_{s}^{2}}\right. \\
& \left.+c_{1} \Lambda_{\text {cut }}^{4}+c_{2} \Lambda_{\text {cut }}^{2} R+\ldots\right],
\end{aligned}
$$

where the ellipses denote the terms of higher adiabatic order. The last two terms in square brackets are the $m_{s}^{2}$ independent "constants" of integration where $c_{1}$ and $c_{2}$ are arbitrary dimensionless constants. 


\subsection{Spinor fields}

The contribution of fermions to the effective action may be calculated in a similar way. Taking a derivative with respect to $m_{\mathrm{F}}$ of the expression (13), in which the action $S^{(2)}$ is replaced by

$$
S_{\mathrm{F}}^{(2)}=\int d^{4} x \sqrt{-g} \mathcal{L}_{\mathrm{F}}^{(2)},
$$

with the Lagrangian (16), we find

$$
\frac{\partial \Gamma_{\mathrm{F}}}{\partial m_{\mathrm{F}}}=\frac{i}{2} \int d^{4} x \sqrt{-g} \operatorname{tr} \mathcal{S}(x, x)
$$

where the trace is taken over the spinor indices. The Feynman Green function $\mathcal{S}$ defined as

$$
\mathcal{S}_{a b}(x, y) \equiv-i<T \Psi_{a}(x) \bar{\Psi}_{b}(y)>
$$

in the limit $y \rightarrow x$ may be expressed as [25]

$$
\mathcal{S}(x, x)=\int \frac{d^{d} k}{(2 \pi)^{d}}\left(-\gamma^{\mu} k_{\mu}+m_{\mathrm{F}}-i \sqrt{2} \lambda \bar{\pi} \gamma_{5}\right) \mathcal{G}(k),
$$

where, to second adiabatic order,

$$
\mathcal{G}(k)=\left[1+\frac{R}{12} \frac{\partial}{\partial m_{\mathrm{F}}^{2}}\right]\left(k^{2}-m_{\mathrm{F}}^{2}\right)^{-1}
$$

Using this we find

$$
\frac{\partial \Gamma_{\mathrm{F}}}{\partial m_{\mathrm{F}}}=2 i \int d^{4} x \sqrt{-g} \int \frac{d^{n} k}{(2 \pi)^{n}}\left(\frac{m_{\mathrm{F}}}{k^{2}-m_{\mathrm{F}}^{2}}+\frac{m_{\mathrm{F}} R}{12\left(k^{2}-m_{\mathrm{F}}^{2}\right)^{2}}\right) .
$$

Finally, using (31) and (32) and integrating (35) over $m_{\mathrm{F}}$ we obtain

$$
\begin{aligned}
& \Gamma_{\mathrm{F}}=\frac{1}{(4 \pi)^{2}} \int d^{4} x \sqrt{-g}\left[m_{\mathrm{F}}^{2} \Lambda_{\mathrm{cut}}^{2}-\frac{m_{\mathrm{F}}^{4}}{4}-m_{\mathrm{F}}^{2}\left(\frac{m_{\mathrm{F}}^{2}}{2}+\frac{R}{12}\right) \ln \frac{\Lambda_{\mathrm{cut}}^{2}}{m_{\mathrm{F}}^{2}}\right. \\
& \left.+c_{3} \Lambda_{\mathrm{cut}}^{4}+c_{4} \Lambda_{\mathrm{cut}}^{2} R+\ldots\right] .
\end{aligned}
$$

Again, the last two terms in square brackets are the $m_{\mathrm{F}}$ independent "constants" of integration where $c_{3}$ and $c_{4}$ are arbitrary dimensionless constants.

\subsection{All together}

Assembling the boson and fermion contributions, the resulting effective action for each species is given by

$$
\Gamma=\Gamma_{\sigma}+\Gamma_{\pi}+\Gamma_{\mathrm{F}}
$$

as a function of the background fields $\bar{\sigma}$ and $\bar{\pi}$. The background fields are determined from the requirement that the vacuum minimizes the potential (10). One may easily verify that 
the function (10) has two minima: The first one is obviously the trivial minimum at $\bar{\pi}=0$, $\bar{\sigma}=0$ in which case all the effective masses are equal $m_{\sigma}=m_{\pi}=m_{\mathrm{F}}=m$. The second minimum occurs at $\bar{\pi}=0, \bar{\sigma}=-\sqrt{2} m / \lambda$. In this case we find $m_{\sigma}=m_{\pi}=-m_{\mathrm{F}}=m$ and hence, this vacuum breaks the supersymmetry and yields a negative fermion mass. However, we immediately note that the fermion contribution, being a function of $m_{\mathrm{F}}^{2}$, is the same for both solutions. Hence, both solutions yield a cancellation of the mass dependent flat spacetime contributions in (41), i.e., the mass dependent terms that do not vanish in the zero curvature limit in the fermion part $\Gamma_{\mathrm{F}}$ precisely cancel the corresponding terms in the boson parts $\Gamma_{\sigma}$ and $\Gamma_{\pi}$.

Next, we fix the unknown constants $c_{1}$ and $c_{2}$ in (33) and $c_{3}$ and $c_{4}$ in (40) from the following considerations [20]. Evaluating formally the functional integral in (13) over boson and fermion fields we may write the one loop effective action as

$$
\Gamma=2 \Gamma_{s}+\Gamma_{\mathrm{F}}=-2 i \ln \left(\operatorname{det} D_{s}\right)^{-1 / 2}-i \ln \operatorname{det} D_{F}=i \operatorname{tr} \ln D_{s}-i \operatorname{tr} \ln D_{\mathrm{F}},
$$

where $D_{s}$ and $D_{\mathrm{F}}$ are the bilinear operators of the Lagrangians (15) and (16) (with $m_{\pi}=$ $m_{\sigma}=m_{\mathrm{F}}=m$ and $\bar{\pi}=0$ ) defined as

$$
D_{s}=-\square-m^{2}-\xi R
$$

and

$$
D_{\mathrm{F}}=i \tilde{\gamma}^{\mu} \nabla_{\mu}-m
$$

Since the final result should depend on $m$ as a function of $m^{2}$ only, we can simplify the trace of the fermion operator using

$$
\operatorname{tr} \ln D_{\mathrm{F}}(m)=\frac{1}{2} \operatorname{tr} \ln D_{\mathrm{F}}(m) D_{\mathrm{F}}(-m)=\operatorname{tr}\left(-\square-\frac{1}{4} R-m^{2}\right),
$$

where the factor $1 / 2$ in front of the trace has been compensated by a factor of 2 for the two degrees of freedom of the Majorana spinor. Hence, the trace of the fermion operator is just the trace of the scalar operator with $\xi=1 / 4$.

Next, we use (22) to invert the operator $D_{s}$ and calculate the trace using

$$
\operatorname{tr} \ln D_{s}=-\operatorname{tr} \ln \Delta .
$$

Working in normal coordinates, in this equation we may replace $\Delta$ by $\bar{\Delta}$ given by (26) which may be expanded as

$$
\bar{\Delta}=\bar{\Delta}_{0}+\bar{\Delta}_{1} R+\ldots,
$$

where $\Delta_{0}$ is the inverse of $\eta^{\mu \nu} \partial_{\mu} \partial_{\nu}-m^{2}$. Hence, we have

$$
\operatorname{tr} \ln D_{s}=-\operatorname{tr} \ln \left(\bar{\Delta}_{0}+\bar{\Delta}_{1} R+\ldots\right)=-\operatorname{tr} \ln \bar{\Delta}_{0}-\operatorname{tr} \bar{\Delta}_{0}^{-1} \bar{\Delta}_{1} R+\ldots .
$$

The first term on the right-hand side of this equation is the flat spacetime contribution which cancels the similar term from the fermion part owing to (42) and (45). Hence, we only need to evaluate the last term in (48)

$$
\operatorname{tr} \bar{\Delta}_{0}^{-1} \bar{\Delta}_{1} R=\int d^{4} x \sqrt{-g} \int d^{4} x^{\prime} \bar{\Delta}_{0}^{-1}\left(x, x^{\prime}\right) \bar{\Delta}_{1}\left(x^{\prime}, x\right) R
$$


which, with a help of the Fourier transform yields

$$
\operatorname{tr} \bar{\Delta}_{0}^{-1} \bar{\Delta}_{1} R=\int d^{4} x \sqrt{-g} \int \frac{d^{4} k}{(2 \pi)^{4}}\left(\xi-\frac{1}{6}\right) \frac{R}{k^{2}-m^{2}} .
$$

Using the regularized momentum integral (31) we find

$$
2 \Gamma_{s}=i \operatorname{tr} \ln D_{s}=-i \operatorname{tr} \ln \bar{\Delta}_{0}-\frac{1}{16 \pi^{2}} \int d^{4} x \sqrt{-g}\left(\xi-\frac{1}{6}\right)\left(\Lambda_{\text {cut }}^{2}-m^{2} \ln \frac{\Lambda_{\text {cut }}^{2}}{m^{2}}\right) R .
$$

Comparing this result with (33) we find

$$
c_{2}=\frac{1}{6}-\xi
$$

Using (45) the fermion part is given by

$$
\Gamma_{\mathrm{F}}=-i \operatorname{tr} \ln D_{\mathrm{F}}=-\left.i \operatorname{tr} \ln D_{s}\right|_{\xi=1 / 4},
$$

which yields

$$
c_{4}=-\left.c_{2}\right|_{\xi=1 / 4}=\frac{1}{12} .
$$

Finally we find the total contribution to the effective action

$$
\Gamma=-\frac{1}{16 \pi^{2}} \int d^{4} x \sqrt{-g} N \tilde{\xi}\left(\Lambda_{\text {cut }}^{2}-m^{2} \ln \frac{\Lambda_{\text {cut }}^{2}}{m^{2}}\right) R
$$

where

$$
\tilde{\xi}=\xi-\frac{1}{4}
$$

Owing to the cancellation of the first term on the right-hand side of (51) with a similar term in the fermion part we obtain $c_{1}+c_{3}=0$. This secures that all the terms in (40) that do not vanish in the zero curvature limit, precisely equal one half of the corresponding terms in the scalar field expression (33), with an opposite sign. In this way, in the sum (41) these terms precisely cancel as they should, because, as demonstrated in [9], supersymmetry provides a cancellation of all flat-spacetime contributions irrespective of the regularization method one uses.

\section{Energy Momentum Tensor}

The vacuum expectation value of the energy momentum tensor is derived from the effective action as

$$
T_{\mu \nu}^{\mathrm{vac}}=\frac{2}{\sqrt{-g}} \frac{\delta \Gamma}{\delta g^{\mu \nu}},
$$

yielding

$$
T_{\mu \nu}^{\mathrm{vac}}=-\frac{N}{8 \pi^{2}} \tilde{\xi}\left(\Lambda_{\mathrm{cut}}^{2}-m^{2} \ln \frac{\Lambda_{\mathrm{cut}}^{2}}{m^{2}}\right)\left(R_{\mu \nu}-\frac{1}{2} g_{\mu \nu} R\right)
$$


First, we note that we generally do not recover the vacuum form of the energy momentum tensor

$$
T_{\mu \nu}=\rho g_{\mu \nu} .
$$

Clearly, the energy momentum tensor (58) cannot be put in the form (59) unless the metric satisfies

$$
R_{\mu \nu} \propto g_{\mu \nu} R
$$

Among homogeneous geometries, this equation holds only for Minkowski and de Sitter spacetimes.

Now, we consider the case when the starting background is provided by pure gravity with the cosmological constant $\Lambda$. Adding (55) to the Einstein-Hilbert action we obtain the total action

$$
S=\frac{1}{16 \pi G} \int d^{4} x \sqrt{-g}\left[R-\frac{G N \tilde{\xi}}{\pi}\left(\Lambda_{\text {cut }}^{2}-m^{2} \ln \frac{\Lambda_{\text {cut }}^{2}}{m^{2}}\right) R-2 \Lambda\right]
$$

which may be recast in the standard Einstein-Hilbert form

$$
S=\frac{1}{16 \pi G_{\mathrm{eff}}} \int d^{4} x \sqrt{-g}\left(R-2 \Lambda_{\mathrm{eff}}\right) .
$$

Here we have introduced the effective Newton and cosmological constants given by

$$
\frac{G_{\mathrm{eff}}}{G}=\frac{\Lambda_{\mathrm{eff}}}{\Lambda}=\lambda
$$

where

$$
\lambda=\left[1-\frac{G N \tilde{\xi}}{\pi}\left(\Lambda_{\text {cut }}^{2}-m^{2} \ln \frac{\Lambda_{\text {cut }}^{2}}{m^{2}}\right)\right]^{-1} .
$$

More specifically, if we demand homogeneity and isotropy the starting background geometry is de Sitter with the expansion parameter $H=\sqrt{\Lambda / 3}$. Then, the resulting effective background is also de Sitter with the effective expansion parameter $H_{\text {eff }}=\sqrt{\Lambda_{\text {eff }} / 3}$.

Consider next a more general case of gravity with matter or dark energy described by the energy momentum tensor $T_{\mu \nu}$. The total energy momentum tensor is the sum of $T_{\mu \nu}$ and $T_{\mu \nu}^{\mathrm{vac}}$ given by (58). The Einstein field equations then read

$$
\left(R_{\mu \nu}-\frac{1}{2} g_{\mu \nu} R\right)=\frac{G N \tilde{\xi}}{\pi}\left(\Lambda_{\text {cut }}^{2}-m^{2} \ln \frac{\Lambda_{\text {cut }}^{2}}{m^{2}}\right)\left(R_{\mu \nu}-\frac{1}{2} g_{\mu \nu} R\right)-8 \pi G T_{\mu \nu}
$$

which may be written as

$$
\left(R_{\mu \nu}-\frac{1}{2} g_{\mu \nu} R\right)=-8 \pi G \lambda T_{\mu \nu}
$$

This equation may be interpreted as the standard Einstein equations in which either the Newton constant $G$ is renormalized as in (63) or the energy momentum tensor is replaced by the effective one

$$
T_{\mu \nu}^{\mathrm{eff}}=\lambda T_{\mu \nu}
$$


Assuming a general perfect fluid form

$$
T_{\mu \nu}=(\rho+p) u_{\mu} u_{\nu}-p g_{\mu \nu}
$$

the energy density and pressure in comoving coordinates are

$$
\begin{gathered}
\rho=<T_{0}^{0}>, \\
p=\frac{1}{3}<T_{0}^{0}-T_{\mu}^{\mu}>.
\end{gathered}
$$

Obviously, equations (68) $-(100)$ also apply to $T_{\mu \nu}^{\text {eff }}$ defined in (67), with $\rho$ and $p$ replaced by $\rho_{\text {eff }}$ and $p_{\text {eff }}$, respectively. Hence, if the energy density and pressure satisfy an equation of state of a simple form $p=w \rho$, then the effective equation of state is of the same form $p_{\text {eff }}=w \rho_{\text {eff }}$. In particular, if the background satisfies the vacuum equation of state $p_{\mathrm{vac}}=-\rho_{\mathrm{vac}}$, the effective equation of state also describes vacuum. However, if the equation of state were more involved, e.g., in the form of a general function $p=p(\rho)$ then the effective equation of state would be deformed, $p_{\text {eff }}=\lambda p\left(\rho_{\text {eff }} / \lambda\right)$.

In order to make comparison with a similar calculations in which a three-dimensional momentum cutoff has been used [10] (see also [11] and [12] for massless scalars) we specify our result to a spatially flat FRW spacetime and $\xi=0$. From (58), we find the leading contribution to the density and pressure as a function of the expansion rate and acceleration

$$
\begin{gathered}
\rho_{\mathrm{vac}}=-\frac{3 N \Lambda_{\mathrm{cut}}}{32 \pi^{2}} \frac{\dot{a}^{2}}{a^{2}}, \\
p_{\mathrm{vac}}=\frac{N \Lambda_{\mathrm{cut}}}{32 \pi^{2}}\left(\frac{\dot{a}^{2}}{a^{2}}+2 \frac{\ddot{a}}{a}\right) .
\end{gathered}
$$

Obviously, the contribution to the energy density is negative and to the pressure is positive for an accelerated expansion. In contrast, a three-dimensional momentum cutoff regularization yields [10]

$$
\begin{gathered}
\rho=\frac{3 N \Lambda_{\text {cut }}}{24 \pi^{2}} \frac{\dot{a}^{2}}{a^{2}}, \\
p=\frac{N \Lambda_{\text {cut }}}{24 \pi^{2}}\left(\frac{\dot{a}^{2}}{a^{2}}-2 \frac{\ddot{a}}{a}\right) .
\end{gathered}
$$

Here, the density is positive and the pressure is negative if $\dot{a}^{2}<2 a \ddot{a}$. For example, for a de Sitter expansion one finds $p=-\rho / 3$ whereas in the present paper $p_{\text {vac }}=-\rho_{\text {vac }}$. Hence, apart from an irrelevant numerical factor (which may be absorbed in the cutoff) and the relative sign between the expansion and acceleration terms in the pressure, we disagree in the overall sign but we agree in the magnitude of the energy density. In both cases a UV cutoff of the order $m_{\mathrm{Pl}} / \sqrt{N}$ yields a magnitude of the leading term in the energy density of the order $H^{2} m_{\mathrm{Pl}}^{2}$, where $H=\dot{a} / a$. Hence, if we identify the expansion parameter $H$ with the Hubble parameter today, the contribution to $\mathrm{CC}$ is of the phenomenologically acceptable order of magnitude and no fine tuning is needed.

Clearly, the above comparison between results obtained with a three-dimensional momentum cutoff and with the present covariant cutoff regularization does not concern renormalized 
quantities. Instead, our aim is to compare the results of the two approaches to an effective field theory with a physical cutoff [31, and, hence, we do not have bare and renormalized $\Lambda$ and $G$, as in [18, 19] (see also [32]). This approach is in spirit similar to [8] where a discrepancy by a factor of about $10^{120}$ was obtained in an effective theoretical estimate of the vacuum energy density with respect to the value compatible with astrophysical and cosmological observations.

Although our aim is not to fit observational data, it is important to note that one cannot have a pure $H^{2} m_{\mathrm{Pl}}^{2}$ for the vacuum energy without additional contributions. At the phenomenological level, it has been already pointed out that only after including an additive term one can get full compatibility with the expansion and structure formation data [33, 34].

\section{Conclusion}

We have calculated the contribution of supersymmetric fields to vacuum energy in a general curved geometry. In addition to supersymmetric fields we have assumed existence of a fluid obeying the equation of state $p=p(\rho)$. Unlike in flat spacetime, the vacuum fluctuations turn out to be nonzero depending on background metric. Combining effects of both the background fluid and vacuum fluctuations of the supersymmetric field in a self-consistent way, we have found the effective equation of state.

In an expanding FRW universe with $H=\dot{a} / a$, we have found that the leading term in the energy density of vacuum fluctuations is negative and, if we impose a UV cutoff of the order $m_{\mathrm{Pl}}$ its magnitude is of the order $H^{2} m_{\mathrm{Pl}}^{2}$. Hence, the vacuum fluctuations of SUSY fields provide a phenomenologically acceptable contribution to the cosmological constant and no fine tuning is needed. The negative sign of the energy density indicates that the vacuum field fluctuations cannot account for $\mathrm{CC}$ alone, hence, one additional positive contribution, either in the form of $\mathrm{CC}$ or a more general form of dark energy, is needed.

\section{Acknowledgments}

We are grateful to Ilya Shapiro and Joan Sola for useful discussions and comments. This work was supported by the Ministry of Science, Education and Sport of the Republic of Croatia under Contract No. 098-0982930-2864.

\section{References}

[1] A.G. Reiss et al., Astronom. J. 116, 1009 (1998);

S. Perlmutter et al., Astrophys. J. 517, 565 (1999);

S. Perlmutter, M.S. Turner, M.J. White, Phys. Rev. Lett. 83, 670 (1999).

[2] C.L. Bennett et al., Astrophys. J. Suppl. 148, 1 (2003) arXiv:astro-ph/0302207];

D.N. Spergel et al., Astrophys. J. Suppl. 148, 175 (2003) [arXiv:astro-ph/0302209];

H.V.P. Peiris et al., Astrophys. J. Suppl. 148, 213 (2003) arXiv:astro-ph/0302225].

[3] D.N. Spergel et al., Astrophys. J. Suppl. 170, 377 (2007) arXiv:astro-ph/0603449. 
[4] S. Weinberg, "The cosmological constant problems," Talk given at DM 2000, Marina del Rey, California, 23-25 Feb 2000, arXiv:astro-ph/0005265.

[5] N.D. Birrell, P.C.W. Davies, Quantum Fields in Curved Space, Cambridge University Press, Cambridge, 1992.

[6] I.L. Buchbinder, S.D. Odintsov, I.L. Shapiro, Effective action in quantum gravity, IOP Publishing, Bristol, 1992

[7] J. Wess and B. Zumino, Nucl. Phys. B 70 (1974) 39.

[8] S. Weinberg, Rev. Mod. Phys. 61, 1 (1989).

[9] N. Bilić, arXiv:1004.4984 [hep-th].

[10] N. Bilić, Phys. Rev. D 83, 105003 (2011) [arXiv:1104.1349 [hep-th]].

[11] M. Maggiore, Phys. Rev. 83 (2011) 063514 arXiv:1004.1782]

[12] M. S. Sloth, Int. J. Mod. Phys. D19, 2259-2264 (2010). arXiv:1005.3241 [astro-ph.CO]].

[13] I. L. Shapiro, J. Sola, C. Espana-Bonet, P. Ruiz-Lapuente, Phys. Lett. B 574, 149-155 (2003).

[14] B. Guberina, R. Horvat, H. Štefančić, Phys. Rev. D 67, 083001 (2003).

[15] G. Ossola and A. Sirlin, Eur. Phys. J. C 31 (2003) 165.

[16] E. K. Akhmedov, arXiv:hep-th/0204048.

[17] A. A. Andrianov, F. Cannata, P. Giacconi, A. Y. Kamenshchik and R. Soldati, Phys. Lett. B 651 (2007) 306 arXiv:0704.1436.

[18] M. Maggiore, L. Hollenstein, M. Jaccard and E. Mitsou, Phys. Lett. B 704 (2011) 102 arXiv:1104.3797 [astro-ph.CO]].

[19] L. Hollenstein, M. Jaccard, M. Maggiore and E. Mitsou, arXiv:1111.5575 [astro-ph.CO].

[20] F. Sobreira, B.J. Ribeiro, and I. Shapiro, arXiv:1107.2262 [gr-qc]].

[21] K. Kratzert, Annals Phys. 308 (2003) 285-310. [hep-th/0303260].

[22] D. Bailin and A. Love, Supersymmetric Gauge Field Theory and String Theory, IOP Publishing, Bristol and Philadelphia, 1999.

[23] L.E. Parker and D.J. Toms, Phys. Rev. D 31, 10 (1985)

[24] J. Wess and J. Bagger, Supersymmetry and Supergravity, Princeton University Press, Princeton, 1983

[25] L.E. Parker and D.J. Toms, Quantum Field Theory in Curved Spacetime, Cambridge University Press, Cambridge 2009. 
[26] B. Riemann, Abhandlungen der Königlichen Gesellschaft der Wissenschaften zu Göttingen 13 (1868) 133; reprinted and edited by H. Weyl, Springer,Berlin, 1920.

[27] I.L. Shapiro, Class. Quantum Grav. 25, 103001 (2008)

[28] T.S. Bunch and L.E. Parker, Phys. Rev. D 20, 2499 (1979)

[29] M.J. Veltman, Acta Phys. Polon. B 12, 437 (1981)

[30] G. Cynolter, E. Lendvai, arXiv:1002.4490 [hep-ph].

[31] G. P. Lepage, in Proceedings of TASI'89: From Actions to Answers, eds. T. DeGrand and D. Toussaint, World Scientific (1989), hep-ph/0506330.

[32] I. L. Shapiro and J. Sola, JHEP 0202 (2002) 006; A. Babić, B. Guberina, R. Horvat and H. Štefančić, Phys. Rev. D 65 (2002) 085002.

[33] S. Basilakos, M. Plionis, J. Sola, Phys. Rev. D80, 083511 (2009). arXiv:0907.4555 [astro-ph.CO]].

[34] J. Grande, J. Sola, S. Basilakos, M. Plionis, JCAP 1108, 007 (2011). arXiv:1103.4632 [astro-ph.CO]]. 